\title{
Respiratory syncytial virus infection modulates interleukin-8 production in respiratory epithelial cells through a transcription factor-activator protein-1 signaling pathway
}

\author{
YAO ZHOU $^{1 *}$, JIN YANG ${ }^{1,2^{*}}$, HUAN DENG $^{1}$, HONG XU $^{1}$, JIAMIN ZHANG $^{1}$, \\ WEISONG JIN ${ }^{1}$, HAIYAN GAO ${ }^{1}$, FENG LIU ${ }^{1}$ and DEYU ZHAO ${ }^{1}$ \\ ${ }^{1}$ Department of Respiratory Medicine, Nanjing Children's Hospital Affiliated to Nanjing Medical University, \\ Nanjing, Jiangsu 210008; ${ }^{2}$ Department of Pediatrics, Jiangsu Huai'an Maternity and Children's Hospital, \\ Huai'an, Jiangsu 223002, P.R. China
}

Received November 18, 2013; Accepted May 28, 2014

DOI: $10.3892 / \mathrm{mmr} .2014 .2357$

\begin{abstract}
Respiratory syncytial virus (RSV) is a leading cause of respiratory duct infection that can result in severe clinical symptoms, particularly among children under 3 years of age. In the current study, the effect of RSV on airway epithelial cell function and the potential signaling pathways involved were investigated. A549 human airway epithelial cells were infected with RSV at a multiplicity of infection of 1 . After $24 \mathrm{~h}$, interleukin (IL)-8 secretion in the cell supernatant was analyzed. A microarray assay of RSV-infected A549 cells was conducted in order to identify any potential pathways involved, and quantitative polymerase chain reaction was performed to examine mRNA expression levels in these pathways. Electrophoretic mobility shift assays of nuclear transcription factors were conducted for further verification. IL-8 levels increased significantly in the supernatant of RSV-infected A549 cells compared with levels in non-infected cells. Microarray data suggested the involvement of the Toll-like receptor 4 (TLR4) pathway, and mRNA expression levels of genes (MYD88, TRAM and TRIF) involved in this pathway were higher in infected cells. Enhanced synthesis of activator protein-1 (AP-1) was observed. RSV infection of A549 cells may promote IL-8 secretion. In conclusion, the results of the present study indicate that the TLR4 signaling pathway, in conjunction with MYD88, TRAM, TRIF and the transcription
\end{abstract}

Correspondence to: Professor Deyu Zhao or Dr Feng Liu, Department of Respiratory Medicine, Nanjing Children's Hospital Affiliated to Nanjing Medical University, 72 Guangzhou Road, Nanjing, Jiangsu 210008, P.R. China

E-mail: zhaodeyu98@126.com

E-mail: axsliu@163.com

*Contributed equally

Key words: respiratory syncytial virus, A549 cells, interleukin-8, activator protein-1 factor AP-1, may activate immune responses to RSV infection in airway epithelial cells.

\section{Introduction}

Among infants and young children under 5 years of age, acute respiratory infection is the most common cause of morbidity and mortality (1). Globally, respiratory syncytial virus (RSV) is the most common cause of childhood acute lower respiratory infection (ALRI) and a major cause of hospital admissions when it results in severe ALRI. Data suggest that RSV is a significant cause of mortality during childhood due to its role in ALRI (2). Previous studies have demonstrated that severe early RSV bronchiolitis is associated with an increased prevalence of allergic asthma, which can persist into early adulthood (3). RSV has also been implicated in other respiratory illnesses, and can be serious in elderly patients and patients with chronic lung disease or immunological deficiency (4).

Cytokines and chemokines, which are secreted by airway epithelial cells, have been demonstrated to be critical in the regulation of local inflammatory processes in the lungs. Production of proinflammatory cytokines, such as interleukin (IL)-15, MICA and IL-6, increases in epithelial cells following RSV infection (5). A number of the underlying pathways or nuclear transcription factors involved have been shown to be important for the replication and budding of RSV, through regulation by protein kinase $C \delta$, hypoxia-inducible factor- $1 \alpha$, or the nuclear factor (NF)- $\kappa$ B pathway (6). In a live RSV-infected human tracheal epithelial cell line (9HTEo), the mRNA expression levels of Toll-like receptors (TLRs) 1-10 were upregulated compared with levels in cells infected with ultraviolet (UV)-inactivated RSV (7). However, the mechanisms and signaling pathways in RSV-induced reactive airway diseases remain unclear.

Previous studies have indicated that IL- 8 is released in the upper respiratory tract in response to RSV infection $(8,9)$. Studies have suggested that IL-8 levels are correlated with clinical disease severity $(10,11)$ in full-term infants and that IL-8 may lead to a genetic predisposition to asthma (12). Another study demonstrated that exposure to IL-8 induced 
migration and proliferation of airway smooth muscle cells (13). In the current study, the aim was to observe the effects of RSV on secretion function in A549 cells (human type II pulmonary epithelial cells) and to examine the possible mechanisms involved.

\section{Materials and methods}

Viruses and cell lines. The RSV long strain was kindly donated by Professor Hongwei Wang from the Medical School of Nanjing University, Nanjing, China. The A549 cell line was obtained from the Cell Resources Center of Shanghai Institutes for Biological Sciences, Shanghai, China.

Cell culture and infection. The A549 cell line was cultured in Eagle's minimal essential medium (Gibco-BRL, Grand Island, NY, USA) containing $10 \%$ fetal calf serum and $10 \mu \mathrm{g} / \mathrm{ml}$ ampicillin. A549 cells were seeded at a density of $5 \times 10^{4}$ cells/well in compliance with the manufacturer's instructions in 6-well tissue culture plates (Corning Inc., Corning, NY, USA). Cells were maintained until they reached $\sim 70 \%$ confluency. After $6 \mathrm{~h}$ of cell culture, half of the A549 cells were infected with RSV at a multiplicity of infection of 1 . The cells were infected with RSV for $24 \mathrm{~h}$. Infection was confirmed using a light microscope (Olympus, Tokyo, Japan).

Measurement of cytokine production. After $24 \mathrm{~h}$ of infection, the culture supernatants of the RSV-infected and non-infected cells were collected. IL-8 protein levels in the culture supernatants were measured using an enzyme-linked immunosorbent assay (ELISA) (EMD Millipore, Darmstadt, Germany).

Microarray analysis. To identify possible signaling pathways involved in the secretion of IL-8, infected and non-infected cells were subjected to microarray analysis (KangChen Bio-tech, Inc., Shanghai, China). Total RNA from each sample was quantified with a NanoDrop ND-1000 spectrophotometer (Thermo Fisher Scientific, Waltham, MA, USA) and RNA integrity was assessed using standard denaturing agarose gel electrophoresis. Total RNA $(\sim 5 \mu \mathrm{g})$ of each sample was used for labeling and array hybridization according to the following steps: i) Reverse transcription with Superscript ds-cDNA Synthesis kit (Invitrogen, Life Technologies, Carlsbad, CA, USA); ii) ds-cDNA labeling with NimbleGen One-Color DNA Labeling kit; iii) array hybridization using the NimbleGen Hybridization system followed by washing with the NimbleGen Wash Buffer kit (Roche Diagnostics, Basel, Switzerland); and iv) array scanning using the Axon GenePix 4000B Microarray scanner (Molecular Devices, Sunnyvale, CA, USA).

Scanned images were then imported into NimbleScan software (version 2.6; Roche Diagnostics) for grid alignment and expression data analysis. Expression data were normalized through quantile normalization and the Robust Multi-chip Average algorithm in the NimbleScan software. The probe level and gene level were imported into GeneSpring GX software (version 11.5.1; Agilent Technologies, Inc., Santa Clara, CA, USA) for further analysis. Genes with values $\geq 50.0$ in $2 / 2$ samples were selected for data analysis. Differentially expressed genes were identified through fold change filtering.
Pathway analysis was applied to determine the roles of these differentially expressed genes in these biological pathways. Hierarchical clustering was performed to clarify distinguishable gene expression profiling among samples.

Quantitative polymerase chain reaction ( $q P C R)$. For microarray data validation, qPCR was performed as previously described (7). Briefly, first-strand synthesis was performed using an RNA First-Strand Synthesis kit (Roche Diagnostics) with $40 \mathrm{ng}$ of total RNA (Roche Diagnostics). SYBR Green (Roche Diagnostics) PCR was performed for MYD88, TRAM and TRIF according to the manufacturer's instructions. GAPDH was used as an endogenous control. The primer sequences were as follows: MYD88 F, 5'-GATGGTGGTGGTTGTCTCTGAT-3' and R, 5'-GCTGGGGAACTCTTTCTTCATT-3'; TRAM F, 5'-TCA AACCCGGAATAATCTTTGCT-3' and R, 5'-GGGCCGCAT GGGTATAACAG-3'; TRIF F, 5'-GCCAGCAACTTGGAA ATCAGC-3' and R, 5'-GGGGTCGTCACAGAGCTTG-3'; GAPDH F, 5'-AGAAGGCTGGGGCTCATTTG-3' and R, 5'-AGGGGCCATCCACAGTCTTC-3'. Data obtained by qPCR were evaluated using the $2^{-\Delta \Delta \mathrm{Ct}}$ method.

Electrophoretic mobility shift assay (EMSA). EMSAs were performed. AP-1 protein was extracted from the nuclei of the two groups of cells (Vazyme, Piscataway, NJ, USA) according to manufacturer's instructions. The EMSA kit was procured from Pierce (Rockford, IL, USA).

Briefly, an AP-1 consensus oligonucleotide was prepared with the following sequence: F, 5'-CGCTTGATGACTCAG CCGGAA-3' and R, 3'-GCGAACTACTGAGTCGGCCTT-5' (Beyotime, Shanghai, China). The nuclear extracts $(10 \mu \mathrm{g})$ were incubated for $20 \mathrm{~min}$ with the gel shift-binding buffer (Beyotime), prior to the separation of the labeled probe and protein-DNA complexes by electrophoresis on a $6 \%$ polyacrylamide gel. Following the electrophoretic transfer of the bound complexes to a nylon membrane (Amersham, Uppsala, Sweden), the transferred DNA was cross-linked to the membrane. Biotin-labeled DNA was then detected through chemiluminescence using ChemiDoc XRS+ System with Image Lab Software (Bio-Rad, Berkeley, CA, USA).

Statistical analysis. Data were analyzed using SPSS software, version 13.0 (SPSS, Inc., Chicago, IL, USA). Homogeneity of variance F-tests were used to compare the samples, followed by $\mathrm{t}$ - or $\mathrm{t}$-test. $\mathrm{P}<0.05$ was considered to indicate a statistically significant difference. Data are presented as the mean \pm standard deviation (SD).

\section{Results}

Airway epithelial responses to RSV infection. Following RSV infection for $24 \mathrm{~h}$, morphological changes in the A549 cells were detected. Cell fusion was observed with a light microscope, which was considered to indicate RSV infection (Fig. 1A).

Release of IL-8 increases in A549 cells following RSV infection. Following RSV infection for $24 \mathrm{~h}$, the IL-8 concentration in the supernatant of A549 cells was measured. ELISA results 
A

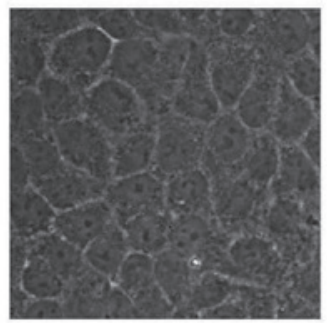

Control

B

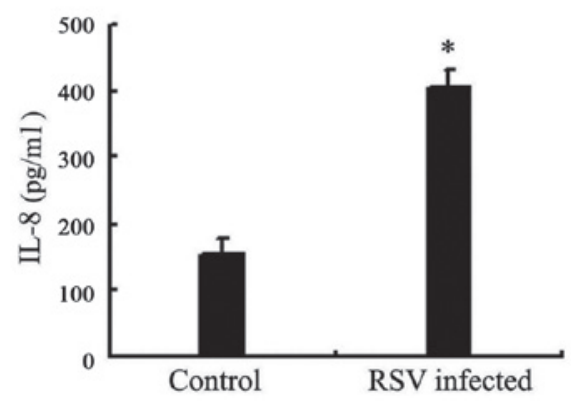

Figure 1. A549 cells exhibit changes in morphology and secretion following infection with RSV. (A) Cell fusion was observed in RSV-infected A549 cells. (B) The levels of IL-8 secretion increased significantly in RSV-infected A549 cells (" $\mathrm{P}<0.05$ vs. non-infected control). RSV, respiratory syncytial virus; IL, interleukin.

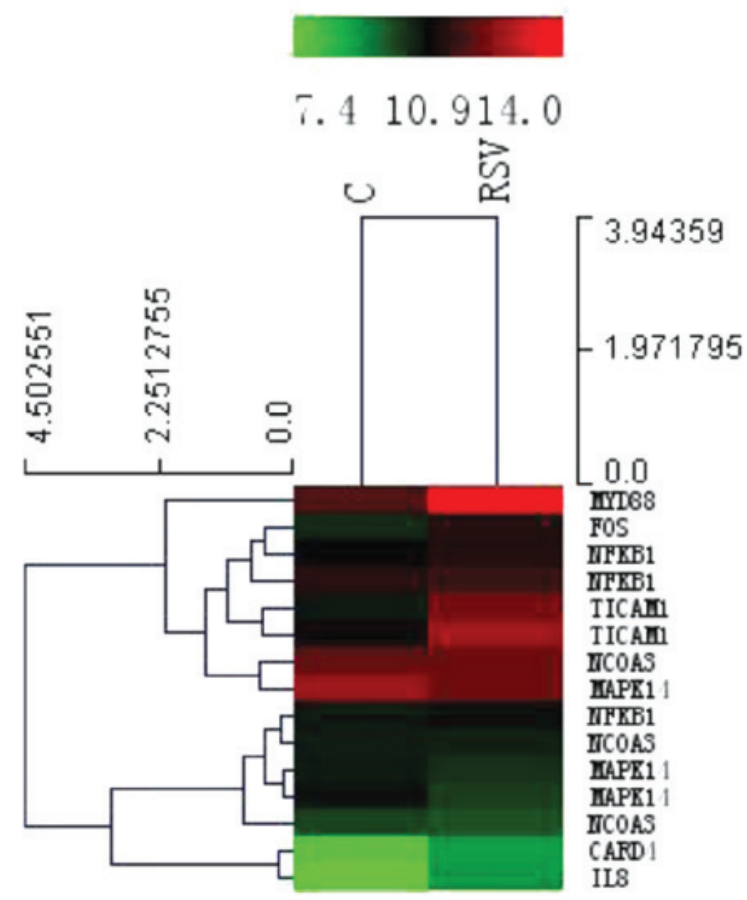

Figure 2. Differential expression of mRNAs between RSV-infected and non-infected A549 cells. RSV, respiratory syncytial virus; C, control.

demonstrated that the IL- 8 concentration in the supernatant of infected cells was $405 \mathrm{ng} / \mathrm{ml}$, while the concentration in non-infected cells was $155 \mathrm{ng} / \mathrm{ml}$, indicating that RSV causes a significant increase $(\mathrm{P}=0.01$; Fig. 1B).

RSV induces changes in mRNA expression levels as detected by microarray analysis. Microarray analysis of human

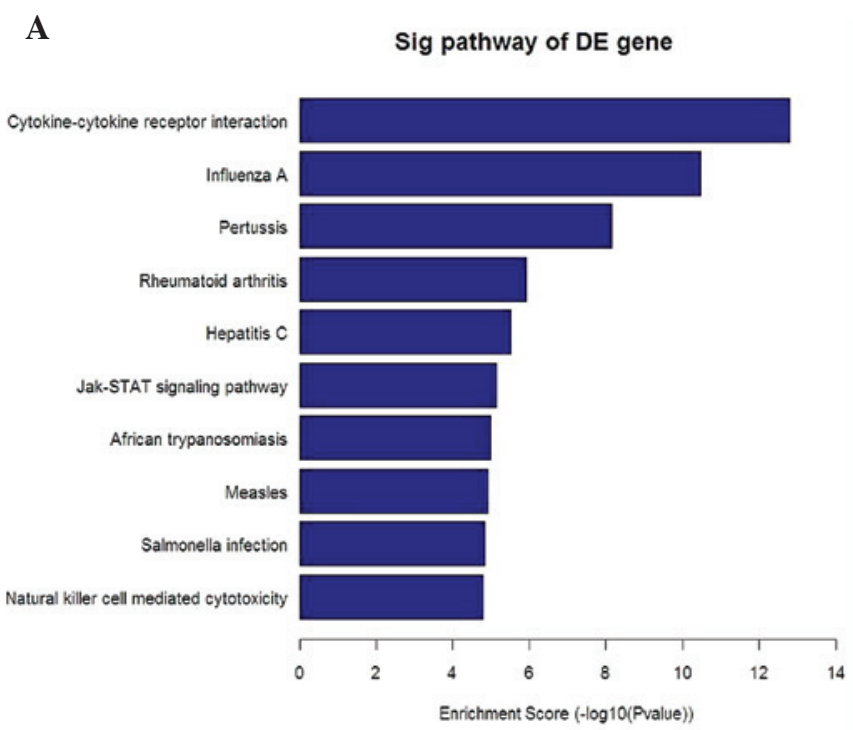

B

Sig pathway of DE gene

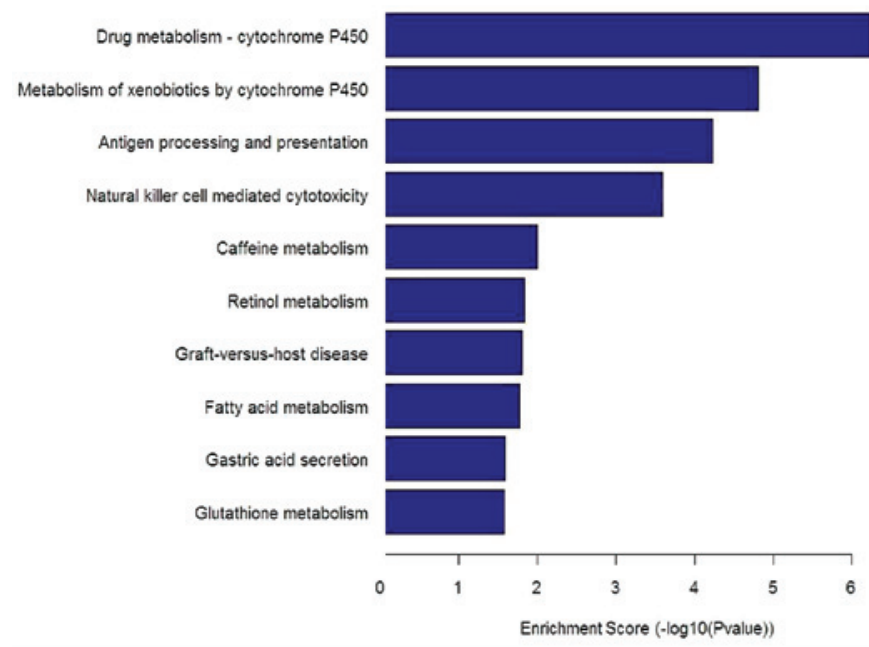

Figure 3. Most significantly enriched signaling pathways (Homo sapiens) involving genes of the miRNAs that were differentially expressed between RSV-infected and non-infected cells. (A) Upregulated and (B) downregulated in the RSV-infected cells vs. the control cells. RSV, respiratory syncytial virus.

bronchial epithelial cells displayed significant changes in the global profile of mRNA expression following infection with RSV. The heat map with hierarchical cluster analysis (Fig. 2) and the bar plots displaying the top ten enrichment score $[-\log 10(\mathrm{P}-\mathrm{value})]$ values of the significantly enriched pathways for each group (Fig. 3A and B) are presented. These imply that the TLR signaling pathways, in particular those of TLR4, are important in the mechanism of IL-8 secretion occurring in RSV-infected A549 cells compared with that in non-infected control cells, in which MYD88, TRAM, TRIF and AP-1 are also involved.

mRNA levels of MYD88, TRAM and TRIF. In order to confirm the effect of the TLR4 signaling pathway on A549 cells following RSV infection, qPCR was performed. mRNA expression levels of MYD88, TRAM and TRIF were significantly increased $(\mathrm{P}=0.035, \mathrm{SD}=0.0116 ; \mathrm{P}=0.026$, 
$\mathrm{SD}=0.00095$; and $\mathrm{P}=0.001, \mathrm{SD}=0.00051$, respectively) in RSV-infected cells compared with those in non-infected cells (Fig. 4).

AP-1 protein expression differences in EMSA. Nuclear factor AP-1 protein expression was investigated by EMSA using nuclear extracts from control and RSV-infected cells at $24 \mathrm{~h}$ post-infection. As shown in Fig. 5, the levels of AP-1 DNA binding in the nuclear fractions were clearly higher in the RSV-infected cells compared with those in the control group. This suggests that RSV infection may induce the activation of AP-1.

\section{Discussion}

Since the primary site for RSV entry and replication is the respiratory tract, the respiratory epithelium has emerged as the major origin of airway inflammation (7). It actively participates in the innate immune response to foreign antigens, which involves the release of chemokines and cytokines, and the initiation of an inflammatory reaction. This is followed by the recruitment of phagocytes, including dendritic cells and lymphocytes, which participate in the clearance of invading pathogens to facilitate the adaptive immune response (9).

In the present study, IL-8 production in A549 human type II pulmonary epithelial cells increased following RSV infection. IL-8 is one of the most abundant chemokines produced by airway epithelial cells. As an important member of the CXC branch of the chemokine family, these proteins may primarily mediate the activation and migration of neutrophils from the peripheral blood into tissue and be involved in the initiation and amplification of inflammatory processes. These processes occur in the human immune system in response to a wide variety of pathogens.

In the current study, the underlying mechanism of the upstream signaling pathway was investigated using microarray experiments and the results indicated that the TLR4 signaling pathway may be partly responsible for increasing the levels of IL-8 secretion. To confirm these results, qPCR and EMSA were conducted for MYD88, TRAM and TRIF, and AP-1, respectively. The results suggested that the transcription factor AP-1 may be important in IL-8 secretion in RSV-stimulated A549 cells.

TLRs are important in the innate immune response, and although they are capable of detecting various molecules derived from viruses, fungi, bacteria and protozoa, intracellular TLRs primarily function in virus detection. All TLRs, with the exception of TLR3, depend to a certain extent on the MyD88 adaptor protein for full signaling activity. TLR4, which is located on the cell surface (14), induces MyD88 signaling at the plasma membrane prior to being endocytosed and subsequently activates TRAM-TRIF signaling from early endosomes (15). When TLR4 migrates to late endosomes, it encounters TAG, a splice variant of TRAM that negatively modulates TLR4-TRAM signaling from endosomes, ultimately promoting the degradation of the signaling complex (16). Activation of interferon regulatory factors, NF- $\mathrm{KB}$ and AP-1 transcription factors induces gene transcription of proinflammatory cytokines such as tumor necrosis factor and IL-12 (17). The AP-1 transcription factor is a dimeric

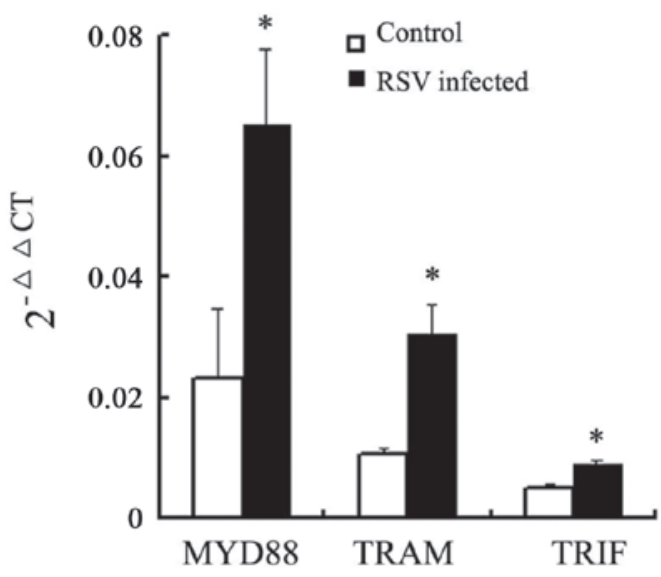

Figure 4. mRNA expression levels of TLR4 signaling pathway genes in RSV-infected A549 cells. Increases in mRNA expression levels in three genes (MYD88, TRAM and TRIF) were detected in RSV-infected cells using the $2^{-\Delta \Delta C t}$ method ( $\mathrm{P}<0.05$ vs. non-infected control cells). RSV, respiratory syncyctial virus; TLR, Toll-like receptor.

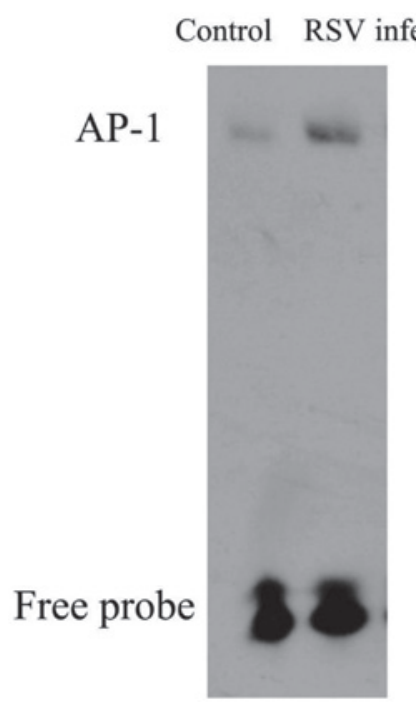

Figure 5. Electrophoretic mobility shift assay exhibiting AP-1 expression changes between RSV-infected and control cells. AP-1 expression increased in RSV-infected compared with that in non-infected A549 cells. RSV, respiratory syncytial virus; AP-1, activator protein-1.

complex that comprises members of the JUN, FOS, activating transcription factor and musculoaponeurotic fibrosarcoma protein families (18). Fos and Jun family proteins function as dimeric transcription factors that bind to AP-1 regulatory elements in the promoter and enhancer regions of numerous mammalian genes. The DNA-binding and dimerization domains of different family members are highly conserved and different members of the Fos and Jun families have similar DNA-binding and dimerization specificities (19). In the process of inflammation, attraction and activity of immune cells are regulated by a plethora of different cytokines that are predominantly activated by the transcription factors, including AP-1. Specifically in the innate immune system, TLRs are an important initiation point of specific sensing for environmental changes. The signaling of TLRs leading to cytokine production may consequently activate AP-1 (20). 
In conclusion, the present study demonstrates one critical signaling pathway in IL-8 secretion of RSV-infected airway epithelial cells, which may be the MyD88/TRAM/TRIF/AP-1 signaling pathway. However, further studies concerning the signaling pathways involved in the increased cytokine production of RSV-infected A549 cells are required.

\section{Acknowledgements}

The current research was supported by the National Natural Science Foundation of China (81200012; Feng Liu), by the Nanjing Medical Science and Technique Development Foundation (Feng Liu), and by a grant from Key Project supported by Medical Science and Technology Development Foundation, Nanjing Department of Health (201108012; Deyu Zhao). The authors also thank the Medical School of Nanjing University for kindly donating RSV, long strain, and Professor Hongwei Wang and Qiuqin Feng for technical support.

\section{References}

1. Liu L, Johnson HL, Cousens S, et al; Child Health Epidemiology Reference Group of WHO and UNICEF: Global, regional, and national causes of child mortality: an updated systematic analysis for 2010 with time trends since 2000. Lancet 379: 2151-2161, 2012.

2. Nair H, Nokes DJ, Gessner BD, Dherani M, Madhi SA, Singleton RJ, O'Brien KL, Roca A, Wright PF, Bruce N, et al: Global burden of acute lower respiratory infections due to respiratory syncytial virus in young children: a systematic review and meta-analysis. Lancet 375: 1545-1555, 2010.

3. Sigurs N, Aljassim F, Kjellman B, Robinson PD, Sigurbergsson F, Bjarnason R and Gustafsson PM: Asthma and allergy patterns over 18 years after severe RSV bronchiolitis in the first year of life. Thorax 65: 1045-1052, 2010.

4. Walsh EE: Respiratory syncytial virus infection in adults. Semin Respir Crit Care Med 32: 423-432, 2011.

5. Zdrenghea MT, Telcian AG, Laza-Stanca V, Bellettato CM, Edwards MR, Nikonova A, Khaitov MR, Azimi N, Groh V, Mallia P, et al: RSV infection modulates IL-15 production and MICA levels in respiratory epithelial cells. Eur Respir J 39: 712-720, 2012.

6. Masaki T, Kojima T, Okabayashi T, et al: A nuclear factor- $\kappa \mathrm{B}$ signaling pathway via protein kinase $C \delta$ regulates replication of respiratory syncytial virus in polarized normal human nasal epithelial cells. Mol Biol Cell 22: 2144-2156, 2011.
7. Xie XH, Law HK, Wang LJ, Li X, Yang XQ and Liu EM: Lipopolysaccharide induces IL-6 production in respiratory syncytial virus-infected airway epithelial cells through the toll-like receptor 4 signaling pathway. Pediatr Res 65: 156-162, 2009.

8. Abu-Harb M, Bell F, Finn A, Rao WH, Nixon L, Shale D and Everard ML: IL-8 and neutrophil elastase levels in the respiratory tract of infants with RSV bronchiolitis. Eur Respir J 14: 139-143, 1999.

9. Gern JE, Martin MS, Anklam KA, Shen K, Roberg KA, Carlson-Dakes KT, Adler K, Gilbertson-White S, Hamilton R, Shult PA, et al: Relationships among specific viral pathogens, virus-induced interleukin- 8 , and respiratory symptoms in infancy. Pediatr Allergy Immunol 13: 386-393, 2002.

10. Hornsleth A, Loland L and Larsen LB: Cytokines and chemokines in respiratory secretion and severity of disease in infants with respiratory syncytial virus (RSV) infection. J Clin Virol 21: 163-170, 2001.

11. Assefa D, Amin N, Dozor AJ and Parton LA: Attenuated interleukin-8/leukocyte immunoresponse in preterm infants compared with term infants hospitalized with respiratory syncytial virus bronchiolitis: a pilot study. Hum Immunol 72: 708-711, 2011.

12. Puthothu B, Krueger M, Heinze J, Forster J and Heinzmann A: Impact of IL8 and IL8-receptor alpha polymorphisms on the genetics of bronchial asthma and severe RSV infections. Clin Mol Allergy 4: 2, 2006.

13. Govindaraju V, Michoud MC, Ferraro P, Arkinson J, Safka K, Valderrama-Carvajal $\mathrm{H}$ and Martin JG: The effects of interleukin-8 on airway smooth muscle contraction in cystic fibrosis. Respir Res 9: 76, 2008.

14. Toshchakov V, Jones BW, Perera PY, Thomas K, Cody MJ, Zhang S, Williams BR, Major J, Hamilton TA, Fenton MJ and Vogel SN: TLR4, but not TLR2, mediates IFN-beta-induced STAT1alpha/beta-dependent gene expression in macrophages. Nat Immunol 3: 392-398, 2002.

15. Kagan JC, Su T, Horng T, Chow A, Akira S and Medzhitov R: TRAM couples endocytosis of Toll-like receptor 4 to the induction of interferon-beta. Nat Immunol 9: 361-368, 2008.

16. Palsson-McDermott EM, Doyle SL, McGettrick AF, Hardy M, Husebye H, Banahan K, Gong M, Golenbock D, Espevik T and O'Neill LA: TAG, a splice variant of the adaptor TRAM, negatively regulates the adaptor MyD88-independent TLR4 pathway. Nat Immunol 10: 579-586, 2009.

17. Blasius AL and Beutler B: Intracellular toll-like receptors. Immunity 32: 305-315, 2010.

18. Eferl R and Wagner EF: AP-1: a double-edged sword in tumorigenesis. Nat Rev Cancer 3: 859-868, 2003.

19. Curran T and Franza BR Jr: Fos and Jun: the AP-1 connection. Cell 55: 395-397, 1988

20. Kawai T, Sato S, Ishii KJ, Coban C, Hemmi H, Yamamoto M, Terai K, Matsuda M, Inoue J, Uematsu S, et al: Interferon-alpha induction through Toll-like receptors involves a direct interaction of IRF7 with MyD88 and TRAF6. Nat Immunol 5: 1061-1068, 2004. 